\section{Caracterización final y lecciones de la epidemia de dengue 3 en Cuba, 2001-2002}

\author{
María G. Guzmán, ${ }^{1}$ Otto Peláez, ${ }^{2}$ \\ Gustavo Kourí, ${ }^{1}$ \\ Ibrahim Quintana, ${ }^{3}$ \\ Susana Vázquez, ${ }^{1}$ \\ Macdelín Pentón, ${ }^{2}$ \\ Luis Carlos Ávila² \\ y el Grupo Multidisciplinario para \\ el Control de la Epidemia \\ de Dengue 2001-20024
}

Palabras claves: dengue, fiebre dengue hemorrágica, vigilancia epidemiológica, brotes de enfermedades, Cuba.

\footnotetext{
1 Centro Colaborador OPS/OMS para el Estudio del Dengue y su Vector, Instituto de Medicina Tropical Pedro Kourí, Ciudad de La Habana, Cuba. La correspondencia debe enviarse a María G. Guzmán, Departamento de Virología, Instituto de Medicina Tropical Pedro Kourí, Autopista Novia del Mediodía km 61/2, Apartado postal Marianao 13, Ciudad de La Habana, Cuba. Correo electrónico: lupe@ ipk.sld.cu

2 Centro Provincial de Higiene y Epidemiología, Ciudad de La Habana, Cuba.

3 Instituto Nacional de Higiene y Epidemiología, Ciudad de La Habana, Cuba.

4 Instituto de Medicina Tropical Pedro Kourí, Ciudad de La Habana, Cuba: Delfina Rosario, Daniel González, Osvaldo Castro, Rosmari Rodríguez, Mailing Álvarez, Juan Bisett, Omar Fuentes, Manuel Díaz, José L. Pelegrino y Eric Martínez; Centro Provincial de Higiene y Epidemiología, Ciudad de La Habana, Cuba: Eugenio Córdova y Ana María Masa; Ministerio de Salud Pública, Ciudad de La Habana, Cuba: Juan Vázquez; Organización Panamericana de la Salud, Programa Regional de Dengue, Ciudad de Panamá, Panamá: José L. San Martín.
}

En los últimos 10 años se ha constatado la reintroducción y expansión del serotipo 3 del virus del dengue en la Región de las Américas. Este serotipo se asoció con las epidemias de 1962-1963 y 19681969 en varios países del Caribe y los últimos aislamientos realizados coincidieron con el momento de entrada del serotipo 1 en la Región en 1977 (1). En 1994 se comprobó la reintroducción del virus del dengue 3, primero en América Central y posteriormente en México, el Caribe y América del Sur (2-4). La cepa introducida entonces difería genéticamente de la que circuló durante las décadas de 1960 y 1970. Varios autores han confirmado que las cepas de dengue 3 aisladas en nuestra Región desde 1994 hasta la fecha pertenecen al genotipo III. Este genotipo originalmente agrupaba cepas aisladas en grandes epidemias ocurridas durante la década de 1980 en la India y Sri Lanka. Los estudios filogenéticos que se han realizado demuestran el origen asiático de la cepa de dengue 3 introducida en 1994, lo que pudiera indicar que ocurrió una evolución in situ de dicha cepa (4-7). Entre los años 2002 y 2005, este serotipo siguió circulando en varios países de la Región de las Américas y provocó graves epidemias $(8,9)$.

A pesar del intenso programa de control y erradicación del principal vector, el mosquito Aedes aegypti, que se aplica en Cuba desde hace más de 20 años, el país no ha estado exento de la reintroducción del virus del dengue. No obstante, la vigilancia activa y las acciones de control establecidas han permitido detectar tempranamente la presencia del virus, evitar grandes brotes y que el dengue se convierta en una enfermedad endémica en la isla (10-12).

En el presente trabajo se describen las principales características clínico-epidemiológicas de la epidemia de dengue 3 que afectó a la República de Cuba en los meses de junio de 2001 a marzo de 2002, así como las acciones establecidas para su eliminación.

\section{ANTECEDENTES}

\section{Características demográficas de Cuba}

La República de Cuba tiene una población de 11230076 (2003) y una densidad poblacional de 101,3 habitantes $/ \mathrm{km}^{2}$, de los cuales 8507406 viven en zonas urbanas (densidad urbana: 4 098,4 habitantes $/ \mathrm{km}^{2}$ ). Desde el punto de vista políticoadministrativo, Cuba está dividida en 14 provin- 
cias, 169 municipios y un municipio especial (Isla de la Juventud). La capital, Ciudad de La Habana, se encuentra al noroeste de la isla con una extensión de 720,84 km² y una población de 2193848 habitantes distribuidos en 15 municipios (densidad de población: 3040 habitantes $/ \mathrm{km}^{2}$ ). La temperatura promedio anual es de $25^{\circ} \mathrm{C}$.

Los centros de salud de la atención primaria constituyen la estructura física y funcional en la que se desarrolla una parte importante de las actividades de la atención primaria de salud en Cuba. Estos centros están integrados por los médicos de familia organizados en torno a la policlínica de cada zona, en la que se planifican, organizan, desarrollan, controlan y evalúan las acciones de promoción, prevención, recuperación y rehabilitación de la salud de las personas, las familias y la comunidad en un espacio geodemográfico determinado: el área de salud.

\section{Antecedentes del dengue en Cuba}

En el período de 1977 a 2000 en Cuba ocurrieron tres epidemias de dengue y un pequeño brote. Las epidemias de 1977 (dengue 1) y 1981 (dengue 2) afectaron a todo el país, mientras que la epidemia de 1997 (dengue 2) se localizó en el municipio de Santiago de Cuba, en la zona oriental de la isla. En las dos últimas epidemias se notificaron casos de dengue hemorrágico (10, 13-15). En septiembre de 2000 se detectó un pequeño brote en tres áreas de salud de Ciudad de La Habana, con 138 casos confirmados de dengue causado por los virus del dengue 3 y $4(12,14)$.

\section{Factores de riesgo}

Entre los factores de riesgo que favorecieron la reintroducción del dengue en Cuba en el año 2001 se encontraban: a) la elevada transmisión de dengue en la región; b) el aumento en el número de personas que viajaban a Cuba desde países endémicos; c) el aumento de la infestación con el vector en varias ciudades del país, principalmente de las provincias Ciudad de La Habana, Santiago de Cuba y Guantánamo.

Además, la provincia Ciudad de La Habana contaba con factores de riesgo específicos que favorecieron la introducción del dengue: la elevada densidad de la población; el hecho de que la ciudad es el sitio de entrada principal de viajeros internacionales y el lugar principal de permanencia hasta su partida al destino final, además del sitio principal donde se desarrollan las actividades culturales, sociales, políticas y deportivas en que participa un gran número de visitantes de países con dengue endémico; y la reinfestación de algunas áreas de la ciudad con el vector $A$. aegypti.

\section{Vigilancia clínica y epidemiológica con apoyo de laboratorio}

La vigilancia del dengue en Cuba se basa en cuatro pilares fundamentales: la clínica, la epidemiología, la entomología y la virología. Su objetivo es buscar, notificar y confirmar expeditamente los casos que clínicamente despiertan la sospecha de que son de dengue $(12,15)$. Ante la presencia de un caso sospechado se desencadenan acciones intensivas de vigilancia activa y de control con la participación tanto del sector de la salud como de otros sectores de la comunidad (16).

En el momento de esta epidemia, el país contaba con tres laboratorios provinciales (Santiago de Cuba, Guantánamo y Ciudad de La Habana) con la capacidad para realizar el diagnóstico serológico del dengue. La confirmación quedó a cargo del Centro Nacional de Referencia, en el Instituto de Medicina Tropical Pedro Kourí $(17,18)$. Las muestras procedentes de las provincias que no disponían de laboratorios para realizar el tamizaje serológico inicial se enviaron directamente al Centro Nacional de Referencia para su estudio.

\section{LA EPIDEMIA}

\section{Detección del brote}

El 29 de junio de 2001, el sistema de vigilancia confirmó la presencia de un caso de dengue en el municipio Playa de Ciudad de La Habana. Los síntomas habían comenzado el 16 de junio. El cuadro clínico se caracterizó por fiebre, mialgias, artralgias, postración y diarrea líquida. Los estudios epidemiológicos y serológicos realizados permitieron averiguar que la introducción viral ocurrió entre el 19 y el 30 de mayo (12).

\section{Fortalecimiento de la vigilancia clínica y epidemiológica con apoyo de laboratorio}

Una vez detectada la transmisión, se alertó a las autoridades nacionales de salud y se intensificó la búsqueda de casos con fiebre de causa no precisada y de casos sospechados de dengue (casos con fiebre y al menos dos síntomas de dengue, como artralgia, mialgia, cefalea o erupción cutánea).

Durante las primeras ocho semanas de la epidemia, cuando el número de casos era pequeño, el 
CUADRO 1. Sueros de casos febriles y con sospecha de dengue evaluados por provincia. Cuba, enero de 2001 a marzo de 2002

\begin{tabular}{lcrl}
\hline \multicolumn{1}{c}{ Provincia } & $\begin{array}{c}\text { Muestras } \\
\text { estudiadas }\end{array}$ & \multicolumn{2}{c}{$\begin{array}{c}\text { Muestras } \\
\text { confirmadas }(\%)\end{array}$} \\
\hline Pinar del Río & 4927 & 194 & $(3,9)$ \\
La Habana & 7057 & 508 & $(7,2)$ \\
Ciudad de La Habana & 83873 & 12889 & $(15,4)$ \\
Matanzas & 2533 & 10 & $(0,4)$ \\
Cienfuegos & 4420 & 17 & $(0,4)$ \\
Sancti Spiritus & 4120 & 14 & $(0,3)$ \\
Villa Clara & 1741 & 5 & $(0,3)$ \\
Ciego de Ávila & 2160 & 17 & $(0,8)$ \\
Camagüey & 1045 & 13 & $(1,2)$ \\
Las Tunas & 1993 & 207 & $(10,4)$ \\
Holguín & 2288 & 10 & $(0,4)$ \\
Granma & 1900 & 6 & $(0,3)$ \\
Santiago de Cuba & 5409 & 86 & $(1,6)$ \\
Guantánamo & 5390 & 548 & $(10,2)$ \\
Isla de la Juventud & 68 & 0 & $(0)$ \\
$\quad$ Total & 128924 & 14524 & $(11,3)$ \\
& & &
\end{tabular}

objetivo de la vigilancia era detectar si aparecían casos de dengue en nuevas áreas de salud y municipios, por lo que la confirmación de los casos se hacía mediante pruebas de detección de anticuerpos específicos de las clases IgM e IgG en sueros recolectados al $5 .^{\circ}$ ó $6 .^{\circ}$ día del comienzo de la fiebre o en sueros pareados. Una vez que el número de enfermos aumentó, se consideraron casos confirmados aquellos en que se detectaba la presencia de anticuerpos IgM.

Cuando se confirmó la transmisión viral en otras provincias del país, se extendió el diagnóstico serológico. Para ello se organizó el diagnóstico en seis laboratorios más mediante un ensayo enzimático ultramicroanalítico de captura de anticuerpos IgM específicos contra el virus del dengue (UMELISA, Centro de Inmunoensayo, Cuba). Tres de esos laboratorios se ubicaron en Ciudad de La Habana y el resto en las provincias de Pinar del Río, Villa Clara y las Tunas (18).

Como parte del trabajo de vigilancia epidemiológica del dengue, en el año 2001 se procesaron 128924 muestras de suero en todo el país y se confirmó la infección por dengue en 11,3\% de los casos. El 65\% de las muestras procedían de Ciudad de La Habana (cuadro 1).

Además de la vigilancia serológica, se estableció la vigilancia virológica y molecular, para lo cual se recolectaron muestras de suero en el plazo de cinco días después de la aparición de los síntomas de dengue y de dengue hemorrágico. Las muestras se colectaron al principio, a mediados y al final de la epidemia y se utilizaron para realizar el aislamiento viral en células de mosquito A. albopictus (C636/HT) y la detección de ácido nucleico viral mediante la reacción en cadena de la polimerasa (19-21). En total se realizaron 91 aislamientos del virus del dengue 3 a partir de las muestras obtenidas de casos de dengue y de dengue hemorrágico. Los estudios moleculares demostraron que la cepa circulante pertenecía al genotipo III (22), previamente asociado con epidemias de dengue hemorrágico en el sudeste Asiático y en nuestra Región (4-7).

\section{Evolución del brote}

A pesar de su temprana detección y de las medidas establecidas para su control, el brote se extendió paulatinamente por otros municipios de Ciudad de La Habana debido al inevitable desplazamiento de la población y a la presencia del vector. En la semana epidemiológica número 29 de ese año - es decir, cuatro semanas después de la detección inicial de la epidemia - ya se había detectado la transmisión en el municipio capitalino de Arroyo Naranjo y para la semana 35 se informaban de casos autóctonos en los municipios 10 de Octubre, Plaza, Marianao, Centro Habana y Cerro. Finalmente, en la semana 38, el brote se extendió a cinco nuevos municipios (Habana Vieja, San Miguel del Padrón, Boyeros, La Lisa y Habana del Este) y en las semanas 41 y 42, a Guanabacoa y Regla, respectivamente. De los 15 municipios de la ciudad, solo en el municipio Cotorro no se detectó la transmisión de la infección, posiblemente por tener un bajo índice de infestación con el vector.

Debido al intenso trasiego de personas entre la capital y las otras provincias del país y a la presencia del vector en algunas de ellas, a finales de septiembre y principios de octubre se detectó la transmisión autóctona en las provincias de La Habana, Pinar del Río y Santiago de Cuba. A principios de noviembre la epidemia llegó a la provincia de Guantánamo y en enero de 2002 a Las Tunas. En total en el país se confirmaron 14524 casos, de ellos 12889 (88,7\%) en Ciudad de La Habana, 194 (1,3\%) en Pinar del Río, 508 (3,5\%) en La Habana, 207 $(1,4 \%)$ en las Tunas, $86(0,6 \%)$ en Santiago de Cuba y 548 (3,8\%) en Guantánamo. En el resto de las provincias del país se confirmó un total de 92 enfermos $(0,7 \%)$ que se infectaron en Ciudad de La Habana. Los primeros casos se localizaron en los alrededores de las estaciones de trenes y ómnibus y siempre en las capitales de provincia. La figura 1 muestra la curva de la epidemia de dengue durante el tiempo que duró el brote. Las provincias Ciudad de La Habana, Guantánamo y La Habana tuvieron las mayores tasas de incidencia (figura 2).

A principios del mes de diciembre se observó una franca disminución en el número de enfermos, 
FIGURA 1. Incidencia de casos de dengue (por 100000 habitantes), por semanas. Cuba, junio de 2001 a marzo de 2002

No. de casos confirmados

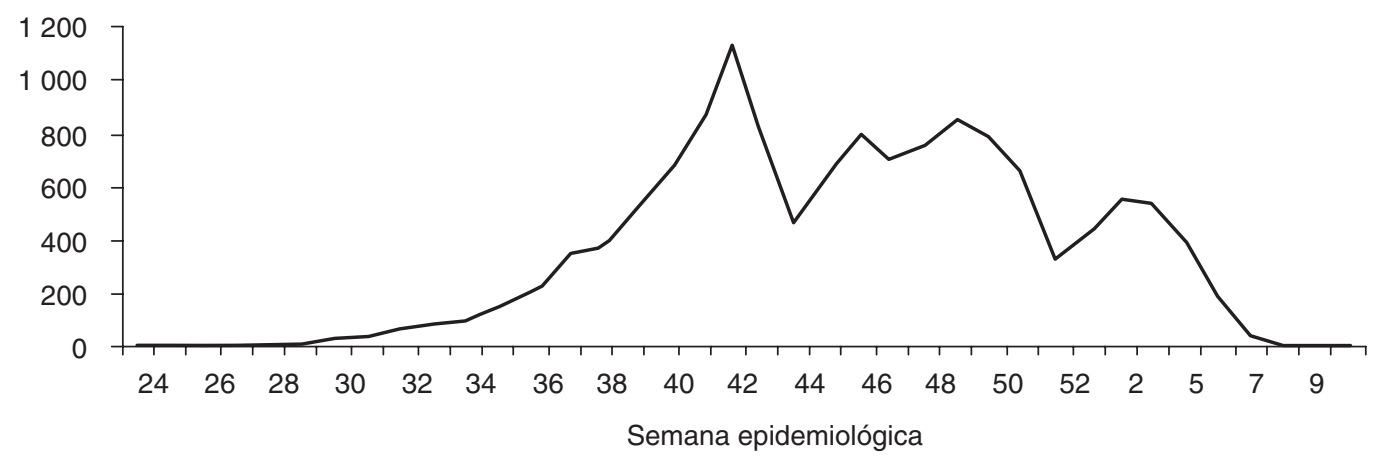

FIGURA 2. Tasas de incidencia de dengue (por 100000 habitantes), por provincias. Cuba, junio de 2001 a marzo de $2002^{\mathrm{a}}$

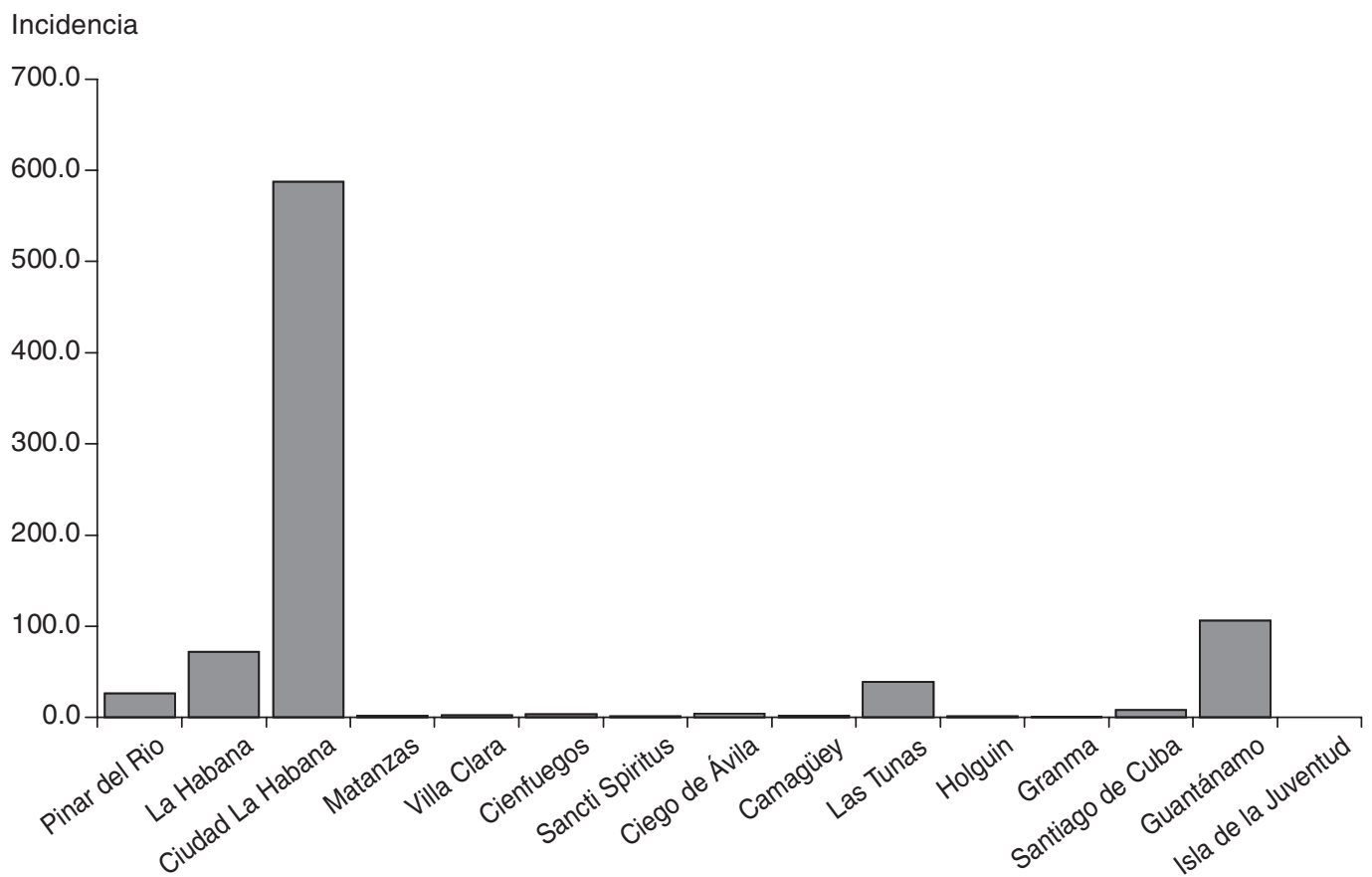

Provincia

a Tasa nacional: 18,1 por 100000 habitantes.

acorde con la disminución en los índices de infestación del vector. Particularmente en Ciudad de La Habana se observó una tendencia descendente en el número de casos confirmados, aunque todavía en algunos municipios esta tendencia era ascendente.

\section{Cuadro clínico}

Para la clasificación de los casos de dengue hemorrágico se creó un comité de expertos integrado por médicos clínicos, virólogos, epidemiólo- gos y patólogos que aplicaron los criterios establecidos en las Guías de la Organización Panamericana de la Salud (OPS) para la Prevención y Control del Dengue (16).

De los 12889 casos confirmados de dengue y dengue hemorrágico en Ciudad de La Habana, $7063(54,8 \%)$ presentaron fiebre como única manifestación clínica, $5748(44,6 \%)$ presentaron el cuadro típico de dengue y $78(0,6 \%)$ las manifestaciones clínicas de dengue hemorrágico. Los síntomas y signos observados en los pacientes con diagnóstico confirmado de dengue se presentan en el cuadro 2. 
CUADRO 2. Principales síntomas y signos presentados por los pacientes con diagnóstico confirmado de dengue. Cuba, junio de 2001 a marzo de 2002

\begin{tabular}{|c|c|c|c|c|c|c|}
\hline \multirow[b]{2}{*}{ Síntomas y signos } & \multicolumn{2}{|c|}{$\begin{array}{c}\text { Ciudad de } \\
\text { La Habana } \\
\text { (12 } 811 \text { casos) }\end{array}$} & \multicolumn{2}{|c|}{$\begin{array}{l}\text { Otras provincias } \\
\text { (1 } 632 \text { casos) }\end{array}$} & \multicolumn{2}{|c|}{$\begin{array}{c}\text { Total } \\
\text { (14 } 443 \text { casos) }\end{array}$} \\
\hline & No. & $\%$ & No. & $\%$ & No. & $\%$ \\
\hline Fiebre & 12811 & 100 & 1612 & 98,7 & 14423 & 99,8 \\
\hline Cefalea & 5185 & 89 & 1355 & 82,9 & 6540 & 45,3 \\
\hline Artralgia & 3460 & 59,4 & 925 & 56,5 & 4385 & 30,4 \\
\hline Mialgia & 1864 & 35,2 & 858 & 52,5 & 2722 & 18,8 \\
\hline Dolor retroocular & 3449 & 59,2 & 866 & 53,0 & 4315 & 29,9 \\
\hline Erupción & 1637 & 28,1 & 439 & 26,9 & 2076 & 14,4 \\
\hline Náuseas o vómitos & 40 & 0,68 & 320 & 19,6 & 360 & 2,5 \\
\hline Dolor abdominal & 21 & 0,36 & 186 & 11,4 & 207 & 1,4 \\
\hline Hemorragia $^{a}$ & 77 & 1,3 & 181 & $11,1^{*}$ & 258 & 1,8 \\
\hline Diarrea & 45 & 0,71 & 124 & 7,6 & 169 & 1,2 \\
\hline Escalofríos & 7 & 0,12 & 181 & 11,1 & 188 & 1,3 \\
\hline Otros & 1235 & 21,2 & 167 & 10,2 & 1402 & 9,7 \\
\hline
\end{tabular}

En total, 1884 de los enfermos eran menores de 15 años; de ellos, 88,2\% vivían en Ciudad de La Habana. Todos los casos graves se presentaron en adultos (cuadro 3). De los pacientes de la capital, $52,4 \%$ (6 747) eran mujeres (tasa: 5,85 por 100000 habitantes) y 47,6\% eran hombres (tasa: 5,90 por 100000 habitantes). En el resto del país, 55\% eran mujeres y el $45 \%$ hombres (tasas: 2,0 y 1,6 por 100000 habitantes, respectivamente).

Del total de casos confirmados en todo el país, $81(0,56 \%)$ se clasificaron como dengue hemorrágico y de ellos tres fallecieron, todos procedentes de Ciudad de La Habana (cuadro 3). En la capital del país, la letalidad por dengue hemorrágico fue de $0,02 \%$, la morbilidad de 5,92 por 100000 habitantes y la mortalidad de 0,13 por 100000 habitantes.

Las principales manifestaciones clínicas observadas en este conjunto de pacientes con dengue hemorrágico confirman lo publicado preliminarmente basado en el estudio de 76 pacientes (23). En todos los casos se comprobó la presencia de trombocitopenia y hemoconcentración y en $23,7 \%$ de los casos se observó choque por dengue. Las edades de los pacientes graves fueron de 16 a 64 años y 51 de ellos eran del sexo masculino $(23,24)$.

La infección secundaria se demostró en el 95\% de los casos de dengue hemorrágico (25). Estos pacientes deben haber desarrollado su infección primaria durante las epidemias de dengue 1 de 1977 ó de dengue 2 de 1981, al menos 20 años antes de la infección secundaria. Esto puede explicar que no se observara ningún caso de dengue hemorrágico en niños, ya que en su mayoría desarrollaron su primera infección por dengue durante esta epidemia, con excepción de los niños que hubieran sufrido la infección primaria durante la epidemia por dengue

CUADRO 3. Distribución por edad de los casos confirmados de dengue y dengue hemorrágico en Ciudad de La Habana y otras provincias. Junio de 2001 a marzo de 2002 ${ }^{a}$

\begin{tabular}{|c|c|c|c|c|c|c|c|}
\hline \multirow{3}{*}{$\frac{\text { Edad (años) }}{\text { Menores de } 5^{\mathrm{b}}}$} & \multicolumn{4}{|c|}{ Ciudad de La Habana } & \multicolumn{3}{|c|}{ Otras provincias } \\
\hline & \multicolumn{2}{|c|}{ Dengue } & \multicolumn{2}{|c|}{$\begin{array}{c}\text { Dengue } \\
\text { hemorrágico }\end{array}$} & \multicolumn{2}{|c|}{ Dengue } & \multirow{2}{*}{$\begin{array}{c}\begin{array}{c}\text { Dengue } \\
\text { hemorrágico }\end{array} \\
0\end{array}$} \\
\hline & 275 & $(221,1)$ & & 0 & 51 & $(6,9)$ & \\
\hline De 5 a 14 & 1387 & $(493,0)$ & & 0 & 171 & $(11,3)$ & 0 \\
\hline De 15 a 59 & 8731 & $(618,0)$ & 76 & $(5,4)$ & 1322 & $(46,3)$ & $3 \quad(0,1)$ \\
\hline 60 ó más & 2496 & $(653,4)$ & 5 & $(1,3)$ & 71 & $(4,5)$ & 0 \\
\hline Total & 12889 & $(585,7)$ & 81 & $(3,7)$ & 1615 & $(17,9)$ & $3 \quad(0,03)$ \\
\hline
\end{tabular}

a Entre paréntesis se ofrece la tasa de incidencia por 100000 habitantes.

b Se notificaron 72 casos en niños menores de un año en Ciudad de La Habana y 5 en otras provincias. 
2 de Santiago de Cuba en 1997. En estos momentos se investiga la secuencia de la infección viral asociada con la forma grave de la enfermedad.

Además de la infección secundaria como factor de riesgo del cuadro grave de la enfermedad, el color blanco de la piel y la anemia de células falciformes se constataron en $83,3 \%$ y $11,1 \%$ de los casos con choque, respectivamente, y el asma bronquial en $18,4 \%$ de los casos de dengue hemorrágico (23).

\section{Control de la epidemia}

Seis meses después de detectada la epidemia, el número de casos de dengue en la capital iba en descenso, pero no se lograba eliminar la transmisión. Ya para esa fecha se habían detectado casos autóctonos en las provincias de La Habana, Pinar del Río, Santiago de Cuba y Guantánamo.

Frente al peligro de que el dengue se estableciera de forma endémica, la máxima autoridad política del país asumió la conducción de las acciones de control y eliminación de la epidemia. El 11 de enero de 2002 se dio inicio a la operación conocida como "Ofensiva contra el enemigo", dirigida a eliminar la transmisión. Con un enfoque multisectorial e interdisciplinario, con una fuerte disciplina y control de las acciones y con la participación de todos los sectores políticos y sociales del país bajo una dirección única, se puso en marcha esta última etapa con el objetivo fundamental de eliminar la transmisión y de controlar el vector mediante la reducción de los criaderos.

Para lograr la reducción de los criaderos $-\mathrm{y}$, consecuentemente, del nivel de infestación con el mosquito adulto - se realizaron intensas campañas de recogida de residuales sólidos. Además, la Unidad Nacional de Vigilancia y Lucha Antivectorial estableció ciclos para el tratamiento focal (cada 12 días) y adulticida (cada 7 días). Como parte de ese proceso, se realizaron batidas en un perímetro de 500 metros alrededor de las viviendas de los enfermos —denominadas "radiobatidas" - y se aplicó temefós (Zellsanid, S.L., España-Cuba) al 1\% en forma de arena a todos los criaderos reales y posibles. También se puso en marcha el plan denominado "autofocal", mediante el cual se promovía que cada familia inspeccionara su casa un día a la semana para la detección y eliminación de criaderos de mosquitos dentro de la vivienda y en su entorno inmediato. Los estudiantes de la enseñanza primaria y secundaria cedieron su descanso de los sábados para participar en la búsqueda de criaderos potenciales y de focos de A. aegypti.

Entre los elementos que llevaron al éxito de esta operación se deben destacar el control de la calidad de las acciones antivectoriales, haber logrado acceder a los locales cerrados para su tratamiento antivectorial y la estrategia de fumigación de la periferia hacia el centro con clorpirifós y cipermetrina (Chemotecnica, Argentina). A fin de evitar los criaderos de vector se sustituyeron los tanques de almacenamiento de agua de la población que no reunían las condiciones necesarias por otros con tapas apropiadas.

En esta etapa, además, los médicos y enfermeros y enfermeras de la familia intensificaron la búsqueda activa de enfermos y se estableció el ingreso intradomiciliario (bajo mosquitero) de los pacientes como medida adicional para evitar la transmisión.

El establecimiento del diagnóstico serológico - crucial para la toma inmediata de las decisionesen tres laboratorios de la capital desempeñó un importante papel en esta etapa.

\section{Fin de la epidemia}

El 22 de marzo de 2002, 30 días después de la fecha de inicio de los síntomas del último caso confirmado, se consideró eliminada la epidemia en todo el país.

Tres elementos fueron decisivos para lograr la eliminación de la epidemia:

a) la participación de toda la población en las actividades de lucha antivectorial, en especial de un elevado número de trabajadores y estudiantes, y la interacción de todos los sectores de la sociedad en el enfrentamiento de la epidemia

b) la participación y utilización de los medios masivos de comunicación que desde el inicio de la campaña informaron e instruyeron a la población acerca de sus objetivos y de las acciones que debían acometerse

c) la participación de la familia en la detección y eliminación de los criaderos reales y potenciales de mosquitos en las viviendas y su entorno inmediato.

Durante los tres meses que duró la "ofensiva", se realizaron entre 5 y 7 ciclos de tratamiento focal y 10 de tratamiento adulticida intradomiciliario. A esto se unió el eficaz tratamiento extradomiciliario con piretroides (Chemotecnica, Argentina) mediante equipos terrestres de arrastre y la aviación; la aplicación a partir de la cuarta fase del tratamiento perifocal residual con Fention (40WP, Bayer, Alemania); y el uso de productos biológicos (Bacillus turingiensis, 1200BTU, LABIOFAM, Cuba) para el control larvario, tanto dentro de las viviendas como en sus alrededores. La combinación de todas estas medidas permitió reducir paulatinamente los índices aédicos (el índice por casa, el ín- 
dice de Breteau y el índice por recipiente) de 0,49 al inicio de la campaña a 0,01 después del quinto ciclo.

\section{LECCIONES PARA EL FUTURO}

Varias lecciones pueden extraerse del estudio de la epidemia cubana de dengue de 2001-2002, entre ellas:

1. La infección secundaria, el color blanco de la piel, la anemia de células falciformes y el asma bronquial fueron factores de riesgo de dengue hemorrágico.

2. Aun en un país con un fuerte programa de vigilancia y control, pueden producirse brotes de dengue con un elevado número de enfermos, incluso si el índice aédico es bajo. Se ha estimado que para evitar la transmisión viral en las condiciones de Cuba, el índice aédico debe ser $<2$. Aunque el índice promedio del vector en Ciudad de La Habana estaba por debajo de ese nivel, en algunas manzanas o cuadras alcanzaba cifras elevadas, lo que unido al movimiento del vector y a la entrada en el país de personas en la fase virémica de la infección pueden haber facilitado la transmisión viral.

3. La activa vigilancia clínica y epidemiológica y el apoyo de laboratorio fueron elementos fundamentales para detectar tempranamente la transmisión y realizar el seguimiento y el estudio de la epidemia. No obstante, es imprescindible que al mismo tiempo se pongan en marcha acciones de control coordinadas, tempranas y eficaces. A pesar de la importancia de la vigilancia clínicoepidemiológica con base de laboratorio, una vez que la transmisión ha sido detectada, el papel principal lo asumen las acciones de control del vector. Teniendo en cuenta la movilidad, tanto del vector como de las personas en la fase virémica de la infección, una vez detectada la transmisión, las acciones de control deben dirigirse no solo a las áreas donde se detectaron los enfermos, sino que deben ampliarse para cubrir la mayor extensión posible a su alrededor, a manera de disminuir primero y posteriormente eliminar la transmisión viral.

4. La estrategia de fumigación de la periferia al centro en cada municipio fue vital para lograr la eliminación del vector y evitar su migración hacia otras áreas cercanas.

5. La búsqueda activa de casos y el ingreso hospitalario o domiciliario bajo mosquitero, unida a las acciones antivectoriales masivas, fue crucial para lograr la eliminación de la transmisión.

6. La amplia participación de la población y de sus diferentes sectores, la participación de los me- dios de difusión bajo una dirección única de mando y una fuerte voluntad política fueron elementos esenciales para lograr la eliminación de la epidemia.

\section{CONCLUSIONES}

La epidemia de dengue que hubo en Ciudad de La Habana y otras provincias entre junio de 2001 y marzo de 2002 demostró una vez más el riesgo que enfrentan los países tropicales de la Región de sufrir epidemias de esta enfermedad, incluso los que cuentan con programas bien establecidos de vigilancia y control. Además, quedó demostrado que si se aplican adecuadamente los principios establecidos por la OPS y la Organización Mundial de la Salud para el control del dengue, se puede eliminar la transmisión y evitar que se convierta en una enfermedad endémica.

La búsqueda y evaluación de nuevos índices vectoriales que reflejen con mayor exactitud el peligro de transmisión (por ejemplo, el índice pupal) y la aplicación de sistemas que permitan estratificar el riesgo de transmisión de dengue en una determinada área (índice por manzana) son acciones de gran prioridad que permitirán mejorar el control y la prevención del dengue.

Finalmente, es importante destacar que los programas de prevención y de control del dengue y de su vector requieren de acciones encaminadas a lograr la sostenibilidad del ordenamiento del medio ambiente como condición previa para mantener bajos los niveles de infestación con el vector.

\section{SYNOPSIS}

\section{Final characterization of and lessons learned from the dengue 3 epidemic in Cuba, 2001-2002}

Over the past 10 years, the American Region has witnessed the reintroduction and dissemination of dengue virus serotype 3. In this paper we describe the main clinical and epidemiologic features of the dengue 3 epidemic that broke out in Cuba between June 2001 and March 2002, as well as the measures that were undertaken to eliminate it. A total of 14524 confirmed cases were reported, 12889 (88,7\%) of them in the City of Havana. Eighty-one cases of dengue hemorrhagic fever were confirmed, three of which died. Secondary infection, white skin color, sickle cell anemia, and bronchial asthma were risk factors for dengue hemorrhagic fever. Active clinical and epidemiologic surveillance and laboratory support were critical to the early detection of transmission and to the monitoring of the epidemic. Widespread involvement of the community and its different sec- 
tors, participation of the mass media with a single leadership in command, and strong political will were the key factors that made it possible to eliminate transmission. This epidemic was one more example of the risk of dengue epidemics faced by countries in the area, including those that have strong surveillance and control programs. It also served to show that by applying the principles established by the Pan
American Health Organization and the World Health Organization for dengue control, transmission can be stopped.

Key words: dengue, dengue hemorrhagic fever, epidemiologic surveillance, disease outbreaks, Cuba.

\section{REFERENCIAS}

1. Morens DM, Rigau-Pérez JG, LópezCorrea RH, Moore CG, Ruiz-Tiben EE, Sather GE, et al. Dengue in Puerto Rico, 1977: public health response to characterize and control an epidemic of multiple serotypes. Am J Trop Med Hyg. 1986;35(1):197-211.

2. Guzmán MG. Dengue type 3 infectionNicaragua and Panama, OctoberNovember, 1994. MMWR. 1995;44:21-4.

3. Guzmán MG, Vázquez $S$, Martínez E, Álvarez M, Rodríguez R, Kourí G, et al. Dengue in Nicaragua, 1994: reintroduction of serotype 3 in the Americas. Bol Oficina Sanit Panam 1996;121(2):102-10.

4. Messer WB, Gubler DJ, Harris E, Sivananthan K, de Silva AM. Emergence and global spread of a dengue serotype 3, subtype III virus. Emerg Infect Dis. 2003; 9(7):800-9.

5. Uzcátegui NY, Comach G, Camacho D, Salcedo M, Cabello de Quintana M, Jiménez M, et al. Molecular epidemiology of dengue virus type 3 in Venezuela. J Gen Virol. 2003;84(Pt 6):1569-75.

6. Guzmán MG, Rosario D, Mune M, Álvarez $M$, Rodríguez $\mathrm{R}$, Kourí G. Relaciones genéticas del virus dengue 3 aislado en la epidemia de FHD en Nicaragua, 1994. Rev Cubana Med Trop. 1996;48(2):114-7.

7. Lanciotti RS, Lewis JG, Gubler DJ, Trent DW. Molecular evolution and epidemiology of dengue-3 viruses. J Gen Virol. 1994;75:65-75.

8. Peyrefitte $\mathrm{CN}$, Pastorino BA, Bessaud M, Gravier P, Tock F, Couissinier-Paris $P$, et al. Dengue type 3 virus, Saint Martin, 2003-2004. Emerg Infect Dis. 2005; 11(5):757-61.

9. de Castro JA, de Andrade HM, do Monte SJ, da Silva AS, Gomes KC, de Brito e Amaral LF, et al. Dengue viruses activity in Piaui, Brazil. Mem Inst Oswaldo Cruz. 2003;98(8):1021-3.
10. Kourí G, Guzmán MG, Valdés L, Carbonel I, Rosario D, Vázquez S, et al. Reemergence of dengue in Cuba: a 1997 epidemic in Santiago de Cuba. Emerg Infect Dis. 1998;4(1):89-92.

11. Kourí G, Mas P, Guzmán MG, Soler M, Goyenechea A, Morier L. Dengue hemorrhagic fever in Cuba, 1981: rapid diagnosis of the etiologic agent. Bull Pan Am Health Organ. 1983;17(2):126-32.

12. Peláez $\mathrm{O}$, Guzmán MG, Kourí G, Pérez $\mathrm{R}$, San Martín JL, Vázquez S, et al. Dengue 3 epidemic, Havana, 2001. Emerg Infect Dis. 2004;10(4):719-22.

13. Kourí GP, Guzmán MG, Bravo JR, Triana C. Dengue haemorrhagic fever/ dengue shock syndrome: lessons from the Cuban epidemic, 1981. Bull World Health Organ. 1989;67(4):375-80.

14. Guzmán MG, Kourí G. Dengue: an update. Lancet Infect Dis. 2002;2(1):33-42.

15. Valdés L, Guzmán MG, Kourí G, Delgado J, Carbonell I, Cabrera MV, et al. Epidemiology of dengue and hemorrhagic dengue in Santiago, Cuba 1997. Rev Panam Salud Publica. 1999;6(1): $16-25$.

16. PAHO. Dengue and dengue hemorrhagic fever in the Americas: guidelines for prevention and control. Washington, D.C.: PAHO; 1994. (Scientific publication No. 548).

17. Vázquez $\mathrm{S}$, Valdés $\mathrm{O}$, Pupo $\mathrm{M}$, Delgado I, Álvarez M, Pelegrino JL, et al. MACELISA and ELISA inhibition methods for detection of antibodies after yellow fever vaccination. J Virol Methods. 2003; 110(2):179-84.

18. Laferte J, Pelegrino JL, Guzmán MG, González G, Vázquez S, Hermida C. Rapid diagnosis of dengue virus infection using a novel 10-microliter IgM antibody capture ultramicroELISA assay (MAC-microELISA dengue). Adv Modern Biotech. 1992;1(19):4.
19. Rodríguez-Roche R, Álvarez M, Guzmán MG, Morier L, Kourí G. Comparison of rapid centrifugation assay with conventional tissue culture method for isolation of dengue 2 virus in C6/36-HT cells. J Clin Microbiol. 2000;38(9):3508-10.

20. Rosario D, Álvarez M, Díaz J, Contreras $\mathrm{R}$, Rodríguez R, Vázquez $\mathrm{S}$, et al. Polymerase chain reaction for rapid detection and serotyping of dengue virus in clinical samples. Rev Panam Salud Publica. 1998;4(1):1-5.

21. Lanciotti RS, Calisher CH, Gubler DJ, Chang GJ, Vorndam AV. Rapid detection and typing of dengue viruses from clinical samples by using reverse transcriptase-polymerase chain reaction. J Clin Microbiol. 1992;30(3):545-51.

22. Rodríguez-Roche R, Álvarez M, Holmes EC, Bernardo L, Kourí G, Gould EA, et al. Dengue virus type 3, Cuba, 2000-2002. Emerg Infect Dis. 2005;11(5).

23. González D, Castro OE, Kourí G, Pérez I, Martínez E, Vázquez S, et al. Classical dengue hemorrhagic fever resulting from two dengue infections spaced 20 years or more apart: Havana, dengue 3 epidemic, 2001-2002. Int J Infect Dis. 2005;9: 280-5.

24. González D, Martínez R, Castro O, Serrano T, Portela D, Vázquez $S$, et al. Evaluation of some clinical, humoral, and imagenological parameters in patients of dengue haemorrhagic fever six months after acute illness. Dengue Bull. 2005;29. (En prensa).

25. Álvarez M, Rodríguez-Roche $R$, Bernardo L, Vázquez S, Morier L, González $D$, et al. Dengue hemorrhagic fever caused by sequential dengue 1-3 infections at a long interval: Havana epidemic, 2001-2002. Am J Trop Med Hyg. 2006. (En prensa). 